\title{
Central Contact for Recruitment
}

National Cancer Institute

\section{Source}

National Cancer Institute. Central Contact for Recruitment. NCI Thesaurus. Code C127523.

The main or principle contact person responsible for coordinating recruitment information for a study. 